\title{
RETRACTED ARTICLE: Phytobacter nematintestini sp. nov., isolated from the intestine of Caenorhabditis elegans and conferring resistance to Bacillus nematocida infection
}

\author{
$\operatorname{Lin~Zhang~}^{1} \cdot$ Xilei Song $^{1} \cdot$ Wenpeng $\mathrm{Li}^{1} \cdot$ Suya Zhao ${ }^{1} \cdot$ Ling Yang $^{2} \cdot$ Min Xia ${ }^{1} \cdot$ Qiuhong Niu ${ }^{1,3}$
}

Received: 20 August 2018 / Accepted: 7 January 2019 / Published online: 9 March 2019

(C) Università degli studi di Milano 2019

The authors have retracted this article [1] because there are fundamental errors in the data presented that undermine the conclusions drawn. All authors agree with this retraction. 1. Zhang, L., Song, X., Li, W. et al. Ann Microbiol (2019). https://doi.org/10.1007/s13213-019-1437-4. The online version of this article contains the full text of the retracted article as electronic supplementary material.

\section{References}

Zhang L, Song X, Li W, Zhao S, Yang L, Xia M, Niu Q (2019) Phytobacter nematintestini sp. nov., isolated from the intestine of Caenorhabditis elegans and conferring resistance to Bacillus nematocida infection. Ann Microbiol. https://doi.org/10.1007/ s13213-019-1437-4

Electronic supplementary material The online version of this article (https://doi.org/10.1007/s13213-019-1437-4) contains supplementary material, which is available to authorized users.

Min Xia

xiamin@nynu.edu.cn

$\triangle$ Qiuhong Niu

qiuhongniu723@163.com

1 Department of Life Science and Biotechnology, Nanyang Normal University, Nanyang 473000, People's Republic of China

2 Nanyang Academy of Agricultural Sciences, Nanyang 473083, Henan, People's Republic of China

3 Henan Key Laboratory of Ecological Security for Water Source Region of Mid-line of South-to-North Diversion Project of Henan Province, Nanyang, People's Republic of China 